\title{
Factor \& Elemental Analysis of Nanotechnology as GreenTechnology using ABCD Framework
}

\author{
P. S. Aithal ${ }^{1} \&$ Shubhrajyotsna Aithal $^{2}$ \\ ${ }^{1}$ Srinivas Institute of Management Studies, Srinivas University, Mangalore - 575001, INDIA \\ ${ }^{2}$ College of Engineering \& Technology, Srinivas University, Mangalore - 574146, India \\ E-mail: psaithal@gmail.com
}

Type of the Paper: Research Paper.

Type of Review: Peer Reviewed.

Indexed in: OpenAIRE.

DOI: https://doi.org/10.5281/zenodo.1451490.

Google Scholar Citation: IJMTS

\section{How to Cite this Paper:}

Aithal, P. S. \& Aithal, Shubhrajyotsna. (2018). Factor \& Elemental Analysis of Nanotechnology as Green Technology using ABCD Framework. International Journal of Management, Technology, and Social Sciences (IJMTS), 3(2), 57-72.

DOI: https://doi.org/10.5281/zenodo.1451490.

International Journal of Management, Technology, and Social Sciences (IJMTS)

A Refereed International Journal of Srinivas University, India.

(C) With Authors.

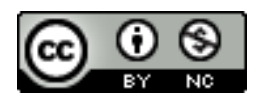

This work is licensed under a Creative Commons Attribution-Non Commercial 4.0 International License subject to proper citation to the publication source of the work.

Disclaimer: The scholarly papers as reviewed and published by the Srinivas Publications (S.P.), India are the views and opinions of their respective authors and are not the views or opinions of the SP. The SP disclaims of any harm or loss caused due to the published content to any party. 


\title{
Factor \& Elemental Analysis of Nanotechnology as Green Technology using ABCD Framework
}

\author{
P. S. Aithal ${ }^{1}$ \& Shubhrajyotsna Aithal $^{2}$ \\ ${ }^{1}$ Srinivas Institute of Management Studies, Srinivas University, Mangalore - 575001, INDIA \\ ${ }^{2}$ College of Engineering \& Technology, Srinivas University, Mangalore - 574146, India \\ E-mail: psaithal@gmail.com
}

\begin{abstract}
There are many definitions for the research process in both natural sciences and philosophical sciences. Out of them, the most popular two definitions are (1) Creating new knowledge, and (2) Interpretation of existing knowledge in a new way through the suitable method of analysis. There are many analysis frameworks used to interpret concepts, ideas, technologies, models, systems, solutions to any problems, strategies, products or services of an organization, resources, etc. Out of them, recently developed analysis framework called ABCD analysis framework is flexible to use in both qualitative and quantitative manner. ABCD analysis method allows the researcher to interpret the given concepts, ideas, technologies, models, systems, solutions of any problems, strategies, products or services of an organization, resources including material, machine, men, money, and information, systematically by considering various determinant issues related to the problem under analysis. The various determinant issues like organizational issue, business issues, customer issues, technological issues, government issue, environmental issues, and societal issues are identified and the affecting factors under the constructs Advantages, Benefits, Constraints, and Disadvantages are determined for identified key attributes. The critical constitutional elements of the nanotechnology as green technology are also identified for the same four constructs. The scores are given to each critical element under all four constructs and based on calculating overall scores the importance of nanotechnology as green technology in solving both basic and advanced problems in the society are determined and rated.
\end{abstract}

Keywords: ABCD Analysis Framework, Green Technology, Factor analysis, Elemental analysis.

\section{INTRODUCTION :}

Technology is an application of science to develop various products and processes, tangible or intangible, to solve problems of the people in the society. Technology provides many business opportunitiesto people where the business is doing anything with profit motivation [1]. There are many different technologies emerged in the society in order to improve the business processes for producing new products or services. The research and developments in science and technology continuously improve the technology or create new technologies. There are many specific-purpose and general-purpose technologies already available and some more are emerging as research progress [2]. Out of many definitions for the research process in both natural sciences and philosophical sciences, the most popular two definitions are (1) Creating new knowledge, and (2) Interpretation of existing knowledge in a new way through the suitable method of analysis. Creating new knowledge mainly focus on creating new science, new technology, new product or service, new process used to produce product or service, creating new way of doing things both theoretically and experimentally. Interpretation of existing knowledge in a new way so that it can support new concept/idea/strategy/solution is also considered as research. For this, a proper analysis tool/framework is required. Such an analysis framework should study and interpret a given 
concept, idea, system, strategy, technology, solution etc. in a systematic form and in detail using various determining issues and affecting factors [3].

Technology analysis is a scientific, interactive, and communicative process that aims to contribute to the formation of public opinion on societal aspects of that technology.There are many analysis frameworks used to interpret concepts, ideas, technologies, models, systems, solutions to any problems, strategies, products or services of an organization, resources, etc. Analysing a technology includes the study of development of that technology, its cost-benefits from the stakeholder's point of view, its strength, weakness, opportunities and challenges (SWOC) [4-5], its advantages, benefits, constraints, and disadvantages to the stakeholders (ABCD) [3, 6], its external analysis using political, economic, social, technological, environmental, and legal (PESTEL) framework [7-8], its performance analysis etc. Out of them, recently developed analysis framework called ABCD analysis framework is flexible to use in both qualitative and quantitative manner [3, 6. 9-12]]. ABCD analysis method allows the researcher to interpret the given concepts, ideas, technologies, models, systems, solutions of any problems, strategies, products or services of an organization, resources including material, machine, men, money, and information, systematically by considering various determinant issues related to the problem under analysis. The various determinant issues like organizational issue, product issue, customer issue, government issue, and environmental/ societal issues are identified and the affecting factors under the constructs Advantages, Benefits, Constraints, and Disadvantages are determined for identified key attributes [5-6]. The ABCD analysing framework is also suitable for analysing a technology to study various affecting factors for different stakeholders including organization which adopted that technology for producing products through suitable processes under given constructs. In this paper, we have used ABCD analysing framework for the first time to analyse nanotechnology which is a general-purpose technology and also a green technology considered as a boon to the mankind due to its usefulness in solving all basic and advanced problems in the society to an optimum level which is very close to the ideal level.

\section{FACTORS \& ELEMENTAL ANALYSIS USING ABCD ANALYSIS :}

The ABCD analysis framework is developed based on two features which include (1) factor analysis and (2) elemental analysis. In factor analysis part, various affecting factors under each determinant issue of the technology are identified for each construct as per a chosen attribute. These affecting factors are identified using the Focus group method. The constructs used in factor analysis are (i) Advantages, (ii) Benefits, (iii) Constraints, (iv) Disadvantages. In the elemental analysis, the critical constitutional elements of the technology are also identified for same determinant issues under same four constructs (i) Advantages, (ii) Benefits, (iii) Constraints, (iv) Disadvantages (ABCD). The scores are given to each critical constituent element under all four constructs and based on calculating overall scores the importance of technology and its constructs in solving both basic and advanced problems in the organization/society are determined and rated [13-25]. In qualitative analysis model,only the affecting factors are determined under same four constructs which is also called ABCD listing [26-37].

\section{NANOTECHNOLOGY AS UNIVERSAL TECHNOLOGY :}

It is known that many emerged technologies found their importance in more than one industry and sector. Such technologies used to create products, process, or services in many areas and to solve many problems or used in many applications in different areas of the society and named as general-purpose technology (GPT) [2]. There are many technologies which have shown such characteristics including those listed in table 1. But recently it is also seen that some of GPT's are grown in such a way that they found applications in solving both basic and advanced problems of the society. The two technologies identified in the $21^{\text {st }}$ century are emerging to such category and hence can be called as 
International Journal of Management, Technology, and Social

universal technologies. They are

Nanotechnology (NT) and Information

Communication and Computation technology

(ICCT). The essential features of such universal technologies are listed in table 2.

Table 1 : Some of identified General Purpose technologies [2]

\begin{tabular}{|l|l|l|l|}
\hline S. No. & Killer Technology & Spill over Effect & Era \\
\hline 1 & Wheel & Mechanization & $4000-3000$ BC \\
\hline 2 & Bronze & Tools \& Weapons & 2800 BC \\
\hline 3 & Printing & Knowledge Economy & $16^{\text {th }}$ Century \\
\hline 4 & Steam Engine & Industrial Revolution & $18^{\text {th }}$ Century \\
\hline 5 & Electricity & Power generation \& Usage & $19^{\text {th }}$ Century \\
\hline 6 & Automobile & $\begin{array}{l}\text { Long distance commuting \& } \\
\text { Transportation }\end{array}$ & $20^{\text {th }}$ Century \\
\hline 7 & Airplane & International Travel \& Transportation & $20^{\text {th }}$ Century \\
\hline 8 & Telephone & Distance communication & $20^{\text {th }}$ Century \\
\hline 9 & Television & Video communication & $20^{\text {th }}$ Century \\
\hline 10 & Computer & Data Processing & $20^{\text {th }}$ Century \\
\hline 11 & Internet & $\begin{array}{l}\text { Data \& Information Communication, E- } \\
\text { business }\end{array}$ & $20^{\text {th }}$ Century \\
\hline 12 & Mobile Communication & Ubiquitous communication & $20^{\text {Th } \text { Century }}$ \\
\hline 13 & Biotechnology & Bio-engineering, Gene Therapy, & $20^{\text {th }}$ Century \\
\hline 14 & $\begin{array}{l}\text { Information } \\
\text { Communication \& } \\
\text { Computation } \\
\text { Technology (ICCT) }\end{array}$ & Ubiquitous computing \& Communication & $21^{\text {st } \text { Century }}$ \\
\hline 15 & $\begin{array}{l}\text { Nanotechnology (NT) } \\
\text { Solutions to nutritious food, drinking } \\
\text { water, renewable energy, Nanomedicine } \\
\text { \& Therapy }\end{array}$ & $21^{\text {st } \text { Century }}$ \\
\hline & $\begin{array}{l}\text { Artificial Intelligence } \\
\text { AI) }\end{array}$ & Total automation & $21^{\text {st } \text { Century }}$ \\
\hline
\end{tabular}

Table 2 : The essential features of Universal technologies

\begin{tabular}{|c|c|c|}
\hline S. No. & Universal Technologies & Major Branches \\
\hline \multirow{10}{*}{1} & \multirow{10}{*}{$\begin{array}{l}\text { Information Communication and } \\
\text { Computation Technology (ICCT) }\end{array}$} & Audio \& Video Communication \\
\hline & & Digital \& Optical computation \\
\hline & & Internet \\
\hline & & Artificial Intelligence \\
\hline & & Big data and business Analytics \\
\hline & & Internet of Things \\
\hline & & Cloud Technology \\
\hline & & Digital Marketing \\
\hline & & 3D Printing \\
\hline & & Virtual Reality \\
\hline \multirow{5}{*}{2} & \multirow{5}{*}{ Nanotechnology (NT) } & Nanobiotechnology \\
\hline & & Nanotechnology for Agricultural Research \\
\hline & & Nanotechnology for Potable water research \\
\hline & & Nanotechnology for Renewable research \\
\hline & & Nanotechnology for Space technology research \\
\hline
\end{tabular}


International Journal of Management, Technology, and Social

\begin{tabular}{|l|l|l|}
\hline \multirow{4}{*}{} & Nanotechnology for Medicine \\
& Nano-pharmacology \\
\cline { 3 - 3 } & Nanoelectronics \\
\cline { 3 - 3 } & Nanophotonics \\
& Nanomechanics \\
\hline
\end{tabular}

\section{NANOTECHNOLOGY AS GREEN} TECHNOLOGY :

Green Technology (GT) can be used for environmental healing that reduces environmental damages created by the conventional industries in the society. It is expected that green technologies reduce the environmental degradation and supports to conserve natural resources. Green technologies are sustainable technologies which will not create a footprint when used for creating various products or processes. Green technologies support the use of natural organic materials and hence do not contribute to produce green gasses which are responsible for environmental degradation. Since green technologies do not support environmental degradation and creating the footprint, they are sustainable and contributes to improve the lifestyle and comfortability of the people.

The nanotechnology emerging as universal technology is expected to solve both basic needs and comfort wants of human beings. The basic needs include food, drinking water, energy, cloth, shelter, health and environment, and the comfort wants are space travel, expanded lifespan etc. Planned and controlled development in nanotechnology leads to environmental sustainability and hence can be used as green technology. Some of the applications of nanotechnology initially thought as green technology are [38] :

- Clean, secure, affordable, renewable energy;

- Stronger, lighter, more durable recyclable materials;

- Low-cost filters to provide clean drinking water from seawater and drainage;

- Medical devices and drugs to detect and treat diseases more effectively with fewer or no side effects;
- Lighting that uses a fraction of the energy associated with conventional systems;

- Sensors to detect and identify harmful chemical and biological agents;

- Techniques to clean up harmful chemicals in the environment.

- Green building and sustainable infrastructure.

- Modified production processes to minimize green gas emission.

The green Nanotechnology is evolved as a general-purpose technology due to its applications in all areas of society and now further growing as universal technology. Hence in the advanced formof universal technology, it will have a significant impact on almost all industries and all areas of society by offering better built, longer lasting, cleaner, safer, and smarter products for the home, for communications, for medicine, for transportation, for agriculture, and for theindustry in general. Thus, by controlled utilization of nanotechnology for environmental sustainability, it can be developed as a green technology for sustainable society.Due to its capability of cleaning of the environmental footprint of other technologies, nanotechnology can be used to enhance the environmentalsustainability of processes currently producing negative externalities. Further nanotechnology can be used to produce various products which can have enhancedenvironmental sustainability. Green nanotechnology has twoobjectives :

(1) The first objective is of producing nanomaterials and fabricating nanoproducts which do not harm the environment and human health and producing nano-products which contribute to solve the environmental problems. It uses many existing principles of green science and green technologyto remove the toxic ingredients of nanomaterials and nano-products used in various industries of the society [39]. 
(2) The second objective of green nanotechnology involves developing nanoproducts that are used to clean the environment either directly or indirectly. In the direct method, nanomaterials or nanoproducts can be used to clean hazardous waste sites, desalinate water, treatment of pollutants, or even sense and monitor any kind of environmental pollutants. In the indirect method, lightweight nanocomposites are used for fabricate automobile and spacecraft parts to save fuel and reduce materials used. Nanotechnology-enabled fuelcells, solar cells, and light-emitting diodes can reduce environmental pollution. Thus, nanotechnology can be used to green the environment for a longtime sustainability [39].

\section{ABCD ANALYSIS OF} NANOTECHNOLOGY :

The widespread growth of nanotechnology as green technology for various business processes can be analysed using many frameworks like SWOC, ABCD, and PESTLE. Out of them, ABCD framework is considered as most appropriate because, it uses many stakeholders as determinant issues. ABCD analysis framework is initially used to analyse systems, ideas, concepts, materials, strategies, and can be also used to analyse technology. Here, we are using ABCD framework to analyse nanotechnology as green technology by considering various determining issues like Organizational issues, Business issues, Consumers issues, Technological issues,
Environmental issues, and Societal issues. Here, green technology features are considered as key attribute to identify the affecting factors under four constructs advantages, benefits, constraints, and disadvantages.

\section{FACTOR ANALYSIS OF NANOTECHNOLOGY :}

The affecting factors under each construct, for identified key attributes for a chosen issue are obtained using focus group method and listed in table 1 . The analysis identified five determinant issues related to nanotechnology as green technology. These include organizational issues, business issues, customer issues, environmental issues, and societal issues. The key attributes identified under organizational issues includes employees of the organizations, infrastructure of the organizations, and the investment of the organizations which involved in nanotechnology based green business. Similarly, the business issues contain four key attributes including products, markets, expansion, and competitors. The customers issues contain four key attributes including quality, features, cost, and support. The Environmental Issues contain two key attributes environmental degradation and Environmental Improvement. Finally, the societal issues contain three key attributes including basic problems, advanced problems, and immortality as given in table 3 . Totally, in this analysis 64 affecting factors are identified and listed under the constructs: advantages, benefits, constraints, and disadvantages.

Table 3:Affecting factors of Nanotechnology as green technology under four constructs

\begin{tabular}{|l|l|l|l|l|l|}
\hline $\begin{array}{l}\text { Determin } \\
\text { ant Issues }\end{array}$ & $\begin{array}{l}\text { Key } \\
\text { Attributes }\end{array}$ & Advantages & Benefits & Constraints & Disadvantages \\
\hline $\begin{array}{l}\text { Organizati } \\
\text { onal Issues }\end{array}$ & Employees & $\begin{array}{l}\text { Skilled } \\
\text { employees }\end{array}$ & $\begin{array}{l}\text { Standard } \\
\text { Quality }\end{array}$ & $\begin{array}{l}\text { Difficulty in } \\
\text { getting } \\
\text { skilled } \\
\text { employees }\end{array}$ & $\begin{array}{l}\text { Cost of } \\
\text { employees }\end{array}$ \\
\cline { 2 - 6 } & Infrastructure & $\begin{array}{l}\text { Less } \\
\text { infrastructure }\end{array}$ & Better return & $\begin{array}{l}\text { New } \\
\text { technology }\end{array}$ & $\begin{array}{l}\text { Risk of new } \\
\text { technology- } \\
\text { based } \\
\text { infrastructure } \\
\text { investment }\end{array}$ \\
\hline
\end{tabular}


International Journal of Management, Technology, and Social

\begin{tabular}{|c|c|c|c|c|c|}
\hline & Investment & $\begin{array}{l}\text { New } \\
\text { technology }\end{array}$ & High return & $\begin{array}{l}\text { Higher } \\
\text { investment }\end{array}$ & $\begin{array}{l}\text { Risk for higher } \\
\text { investment }\end{array}$ \\
\hline \multirow[t]{4}{*}{$\begin{array}{l}\text { Business } \\
\text { Issues }\end{array}$} & Products & $\begin{array}{l}\text { Nanoproducts } \\
\text { can be green } \\
\text { products }\end{array}$ & $\begin{array}{l}\text { Clean } \\
\text { environment }\end{array}$ & $\begin{array}{l}\text { High } \\
\text { production } \\
\text { cost }\end{array}$ & $\begin{array}{ll}\text { May } & \text { have } \\
\text { hidden } & \text { long } \\
\text { term } & \end{array}$ \\
\hline & Markets & $\begin{array}{l}\text { Easy due to } \\
\text { special } \\
\text { characteristics }\end{array}$ & $\begin{array}{l}\text { Better } \\
\text { business }\end{array}$ & $\begin{array}{l}\text { Creating } \\
\text { awareness }\end{array}$ & $\begin{array}{l}\text { Initial Cost of } \\
\text { creating } \\
\text { awareness }\end{array}$ \\
\hline & Competitors & $\begin{array}{lr}\text { Difficult to } \\
\text { copy by } \\
\text { competitors }\end{array}$ & Monopoly & $\begin{array}{l}\text { New } \\
\text { technology }\end{array}$ & $\begin{array}{ll}\text { Huge } & \\
\text { investment for } \\
\text { research \& } \\
\text { development }\end{array}$ \\
\hline & Expansion & $\begin{array}{l}\text { Opportunity } \\
\text { for expansion }\end{array}$ & $\begin{array}{l}\text { Increased } \\
\text { market } \\
\text { share }\end{array}$ & $\begin{array}{l}\text { Educating } \\
\text { the } \\
\text { collaborator } \\
\mathrm{S}\end{array}$ & $\begin{array}{l}\text { Copying by } \\
\text { others }\end{array}$ \\
\hline \multirow{4}{*}{$\begin{array}{l}\text { Consumer } \\
\text { Issues }\end{array}$} & Quality & Optimum & $\begin{array}{l}\text { Durability\& } \\
\text { renewable }\end{array}$ & Cost & $\begin{array}{l}\text { May have side } \\
\text { effects }\end{array}$ \\
\hline & Features & Many & $\begin{array}{l}\text { Comfortable } \\
\text { life }\end{array}$ & $\begin{array}{l}\text { Confused } \\
\text { due to } \\
\text { general } \\
\text { purpose } \\
\text { technology }\end{array}$ & $\begin{array}{l}\text { Fear on Side } \\
\text { effects }\end{array}$ \\
\hline & Cost & Low pollutant & $\begin{array}{l}\text { Helpful for } \\
\text { poor people }\end{array}$ & $\begin{array}{l}\text { Affordabilit } \\
\mathrm{y}\end{array}$ & $\begin{array}{lr}\text { Difficulty } & \text { in } \\
\text { large } & \text { scale } \\
\text { operation } & \\
\end{array}$ \\
\hline & Support & $\begin{array}{l}\text { Low failure } \\
\text { rate }\end{array}$ & $\begin{array}{l}\text { Low } \\
\text { maintenance } \\
\text { cost }\end{array}$ & $\begin{array}{l}\text { Frequent } \\
\text { service }\end{array}$ & $\begin{array}{l}\text { Minimum } \\
\text { service support }\end{array}$ \\
\hline \multirow[t]{2}{*}{$\begin{array}{l}\text { Environm } \\
\text { ental } \\
\text { Issues }\end{array}$} & $\begin{array}{l}\text { Environmenta } \\
\text { l degradation }\end{array}$ & $\begin{array}{l}\text { Less } \\
\text { environmental } \\
\text { degradation }\end{array}$ & $\begin{array}{l}\text { Less } \\
\text { pollution }\end{array}$ & $\begin{array}{l}\text { Complexity } \\
\text { in the } \\
\text { system }\end{array}$ & $\begin{array}{l}\text { Prolonged } \\
\text { technology } \\
\text { breakthrough } \\
\text { than expected }\end{array}$ \\
\hline & $\begin{array}{l}\text { Environmenta } \\
1 \\
\text { Improvement }\end{array}$ & Cleaning & $\begin{array}{l}\text { Better } \\
\text { environment }\end{array}$ & Scaling & $\begin{array}{l}\text { Negative } \\
\text { effects if any }\end{array}$ \\
\hline \multirow[t]{2}{*}{$\begin{array}{l}\text { Social } \\
\text { Issues }\end{array}$} & $\begin{array}{l}\text { Basic } \\
\text { problems }\end{array}$ & $\begin{array}{l}\text { Can be solves } \\
\text { optimally }\end{array}$ & $\begin{array}{l}\text { All basic } \\
\text { problems } \\
\text { will be } \\
\text { solved }\end{array}$ & $\begin{array}{l}\text { Commercial } \\
\text { ization }\end{array}$ & $\begin{array}{ll}\text { Delay in } \\
\text { realization }\end{array}$ \\
\hline & $\begin{array}{l}\text { Advanced } \\
\text { problems }\end{array}$ & $\begin{array}{l}\text { Can be solved } \\
\text { optimally }\end{array}$ & $\begin{array}{l}\text { All } \\
\text { advanced } \\
\text { problems } \\
\text { for human } \\
\text { comfortabili }\end{array}$ & $\begin{array}{l}\text { Still in } \\
\text { experimenta } \\
\text { l level }\end{array}$ & $\begin{array}{l}\text { Unpredictable } \\
\text { adverse effects }\end{array}$ \\
\hline
\end{tabular}

P. S. Aithal. et al. (2018); www.srinivaspublication.com 
International Journal of Management, Technology, and Social

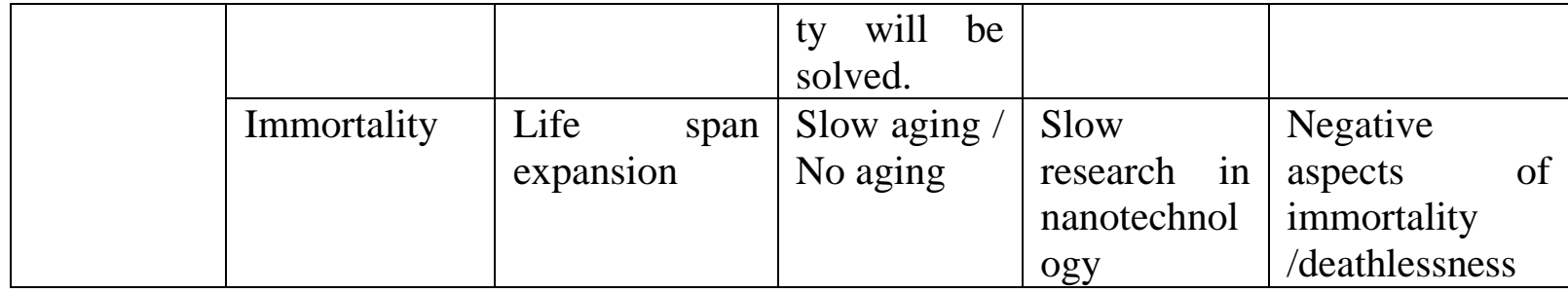

7. ELEMENTAL ANALYSIS OF NANOTECHNOLOGY :

Apart from affecting factors identified in previous section through factor analysis framework, one can also carry out the elemental analysis to identify critical constituent elements for each affecting factorfor chosen determinant issues. These critical constituent elements for the four constructs advantages, benefits, constraints, and disadvantages are listed in tables 4 to 7 respectively.

Table 4 : Advantages of Nanotechnology as Green technology

\begin{tabular}{|c|c|c|c|}
\hline Particulars & Key Attributes & Factors Affecting & Critical Constituent Elements \\
\hline \multirow[t]{3}{*}{$\begin{array}{l}\text { Organizational } \\
\text { Issues }\end{array}$} & Employees & Skilled employees & $\begin{array}{l}\text { (i) Increased productivity } \\
\text { (ii) Sophisticated facility } \\
\text { (iii) More Jobs }\end{array}$ \\
\hline & Infrastructure & Less infrastructure & $\begin{array}{l}\text { (i) Small facilities } \\
\text { (ii) Easy to manufacture } \\
\text { (iii) Decreased cost }\end{array}$ \\
\hline & Investment & New technology & $\begin{array}{l}\text { (i) More initial investment } \\
\text { (ii) New products } \\
\text { (iii) New business }\end{array}$ \\
\hline \multirow[t]{4}{*}{ Business Issues } & Products & $\begin{array}{l}\text { Nanoproducts can be } \\
\text { green products }\end{array}$ & $\begin{array}{l}\text { (i) Low pollution } \\
\text { (ii) Possibility to clean the } \\
\text { environment } \\
\text { (iii) Less side effects }\end{array}$ \\
\hline & Marketing & $\begin{array}{l}\text { Easy due to special } \\
\text { characteristics }\end{array}$ & $\begin{array}{l}\text { (i) Products have ideal characteristics } \\
\text { (ii) Easy to market } \\
\text { (iii) Many positive features }\end{array}$ \\
\hline & Competitors & $\begin{array}{l}\text { Difficult to copy by } \\
\text { competitors }\end{array}$ & $\begin{array}{l}\text { (i) Research for development } \\
\text { (ii) Patented protection } \\
\text { (iii) Uniqueness }\end{array}$ \\
\hline & Expansion & $\begin{array}{l}\text { Opportunity } \\
\text { expansion }\end{array}$ & $\begin{array}{l}\text { (i) Global expansion opportunity } \\
\text { (ii) Opportunity for cost leader } \\
\text { (iii) Opportunity for collaboration }\end{array}$ \\
\hline \multirow[t]{3}{*}{$\begin{array}{l}\text { Consumer } \\
\text { Issues }\end{array}$} & Quality & Optimum & $\begin{array}{l}\text { (i) Ideal products } \\
\text { (ii) Expected quality } \\
\text { (iii)Durability }\end{array}$ \\
\hline & Features & Many & $\begin{array}{l}\text { (i) Green products } \\
\text { (ii) Small \& light in weight products } \\
\text { (iii) Strong products }\end{array}$ \\
\hline & Cost & Low pollutant & $\begin{array}{l}\text { (i) Moderate cost-pollution ratio } \\
\text { (ii) Controlling the pollution } \\
\text { (iii) Low cost high durability }\end{array}$ \\
\hline
\end{tabular}


International Journal of Management, Technology, and Social

\begin{tabular}{|c|c|c|c|}
\hline & Support & Low failure rate & $\begin{array}{l}\text { (i) Durability } \\
\text { (ii) No frequent replacement } \\
\text { (iii) More operation period } \\
\end{array}$ \\
\hline \multirow[t]{2}{*}{$\begin{array}{l}\text { Environmental } \\
\text { Issues }\end{array}$} & $\begin{array}{l}\text { Environmental } \\
\text { degradation }\end{array}$ & $\begin{array}{l}\text { Less environmental } \\
\text { degradation }\end{array}$ & $\begin{array}{l}\text { (i) No contribution to pollution } \\
\text { (ii) Improving environment } \\
\text { (iii) Quality environment for future } \\
\text { generations }\end{array}$ \\
\hline & $\begin{array}{l}\text { Environmental } \\
\text { Improvement }\end{array}$ & $\begin{array}{l}\text { Cleaning } \\
\text { environment }\end{array}$ & $\begin{array}{l}\text { (i) Possibility to clean environment } \\
\text { (ii) Possibility to green the } \\
\text { environment } \\
\text { (iii) Improved products usage }\end{array}$ \\
\hline \multirow[t]{3}{*}{ Social Issues } & Basic problems & $\begin{array}{l}\text { Can be solves } \\
\text { optimally }\end{array}$ & $\begin{array}{l}\text { (i) Optimum to ideal solutions } \\
\text { (ii) Opportunity to solve basic } \\
\text { problems } \\
\text { (iii) }\end{array}$ \\
\hline & $\begin{array}{l}\text { Advanced } \\
\text { problems }\end{array}$ & $\begin{array}{l}\text { Can be solved } \\
\text { optimally }\end{array}$ & $\begin{array}{l}\text { (i) People can live comfortable life } \\
\text { (ii) Life is going to be easy } \\
\text { (iii) Equality for everyone }\end{array}$ \\
\hline & Immortality & Life span expansion & $\begin{array}{l}\text { (i) Disease control } \\
\text { (ii) Health equality } \\
\text { (iii) Enhanced time for achievements }\end{array}$ \\
\hline
\end{tabular}

Table 5 : Benefits of Nanotechnology as Green technology

\begin{tabular}{|c|c|c|c|}
\hline Particulars & Key Attributes & Factors Affecting & Critical Constituent Elements \\
\hline \multirow[t]{3}{*}{$\begin{array}{l}\text { Organizational } \\
\text { Issues }\end{array}$} & Employees & Standard Quality & $\begin{array}{l}\text { (i) Increased return } \\
\text { (ii) Improved working conditions } \\
\text { (iii) More salary }\end{array}$ \\
\hline & Infrastructure & Better return & $\begin{array}{l}\text { (i) Increased profit } \\
\text { (ii) Sustainability } \\
\text { (iii) Further investment opportunity }\end{array}$ \\
\hline & Investment & High return & $\begin{array}{l}\text { (i) More profit } \\
\text { (ii) More return to stakeholders } \\
\text { (iii) Expansion to other areas }\end{array}$ \\
\hline \multirow[t]{4}{*}{ Business Issues } & Products & Clean environment & $\begin{array}{l}\text { (i) Less pollution } \\
\text { (ii) Good health } \\
\text { (iii) Clean resources } \\
\end{array}$ \\
\hline & Marketing & Better business & $\begin{array}{l}\text { (i) Business growth } \\
\text { (ii) Attractive products } \\
\text { (iii)More sales }\end{array}$ \\
\hline & Competitors & Monopoly & $\begin{array}{l}\text { (i) High tech products } \\
\text { (ii) Patenting opportunity } \\
\text { (iii)Enhanced earning } \\
\end{array}$ \\
\hline & Expansion & Increased market share & $\begin{array}{l}\text { (i) More branches } \\
\text { (ii) More subsidiaries } \\
\text { (iii)More products for different } \\
\text { problems }\end{array}$ \\
\hline Consumer & Quality & $\begin{array}{l}\text { Durability } \\
\text { renewable }\end{array}$ & $\begin{array}{l}\text { (i) No environmental pollution } \\
\text { (ii) Clean products }\end{array}$ \\
\hline
\end{tabular}


International Journal of Management, Technology, and Social

\begin{tabular}{|c|c|c|c|}
\hline \multirow[t]{4}{*}{ Issues } & & & (iii) Trouble free operations \\
\hline & Features & Comfortable life & $\begin{array}{l}\text { (i) More essential features } \\
\text { (ii) Need based support } \\
\text { (iii)Availability of essential } \\
\text { components }\end{array}$ \\
\hline & Cost & $\begin{array}{l}\text { Helpful for } \begin{array}{l}\text { poor } \\
\text { people }\end{array} \\
\end{array}$ & $\begin{array}{l}\text { (i) Low cost } \\
\text { (ii) Improved quality of life } \\
\text { (iii) Abundantly available } \\
\end{array}$ \\
\hline & Support & Low maintenance cost & $\begin{array}{l}\text { (i) Quality products } \\
\text { (ii) Self-repairing technology } \\
\text { (iii) Product replacement opportunity }\end{array}$ \\
\hline \multirow[t]{2}{*}{$\begin{array}{l}\text { Environmental } \\
\text { Issues }\end{array}$} & $\begin{array}{l}\text { Environmental } \\
\text { degradation }\end{array}$ & Less pollution & $\begin{array}{l}\text { (i) Clean environment } \\
\text { (ii) Green environment } \\
\text { (iii) Better living conditions }\end{array}$ \\
\hline & $\begin{array}{l}\text { Environmental } \\
\text { Improvement }\end{array}$ & Better environment & $\begin{array}{l}\text { (i) Comfortable life } \\
\text { (ii) Health improvement } \\
\text { (iii) Less diseases }\end{array}$ \\
\hline \multirow[t]{3}{*}{ Societal Issues } & Basic problems & $\begin{array}{l}\text { All basic problems } \\
\text { will be solved }\end{array}$ & $\begin{array}{l}\text { (i) Abundancy in food, water and } \\
\text { energy } \\
\text { (ii) Progressed civilization } \\
\text { (iii)Improved living conditions }\end{array}$ \\
\hline & $\begin{array}{l}\text { Advanced } \\
\text { problems }\end{array}$ & $\begin{array}{l}\text { All advanced problems } \\
\text { for } \\
\text { comfortability will be } \\
\text { solved. }\end{array}$ & $\begin{array}{l}\text { (i) } \begin{array}{l}\text { Self sufficiency in Resources } \\
\text { usage }\end{array} \\
\text { (ii) Decreased social differences } \\
\text { (iii) Opportunities for everybody }\end{array}$ \\
\hline & Immortality & Slow aging / No aging & $\begin{array}{l}\text { (i) No diseases } \\
\text { (ii) Automatic curing of diseases } \\
\text { (iii) Expanded lifespan }\end{array}$ \\
\hline
\end{tabular}

Table 6 : Constraints of Nanotechnology as Green technology

\begin{tabular}{|l|l|l|l|}
\hline Particulars & Key Attributes & Factors Affecting & Critical Constituent Elements \\
\hline \multirow{3}{*}{$\begin{array}{l}\text { Organizational } \\
\text { Issues }\end{array}$} & Employees & $\begin{array}{l}\text { Difficulty in getting } \\
\text { skilled employees }\end{array}$ & $\begin{array}{l}\text { (i) Essential Technology education } \\
\text { (ii) Continuous Research \& training } \\
\text { (iii) Advanced skills }\end{array}$ \\
\cline { 2 - 5 } & Infrastructure & New technology & $\begin{array}{l}\text { (i) Investment for new technology } \\
\text { infrastructure } \\
\text { (ii) Continuous upgradation } \\
\text { (iii) Frequent upgradation }\end{array}$ \\
\cline { 2 - 5 } Business Issues & Investment & Higher investment & $\begin{array}{l}\text { (i) Investment for survival } \\
\text { (ii) Investment for sustainability } \\
\text { (iii) Investment for monopoly }\end{array}$ \\
& Products & High production cost & $\begin{array}{l}\text { (i) Due to spreading of } \\
\text { commercialization cost on production } \\
\text { (ii) Due to complex technology } \\
\text { (iii) Due to advanced features }\end{array}$ \\
\cline { 2 - 4 } & Marketing & Creating awareness & $\begin{array}{l}\text { (i) Training to create awareness } \\
\text { (ii) Acceptability of people } \\
\text { (iii) Education level of people }\end{array}$ \\
\hline
\end{tabular}


International Journal of Management, Technology, and Social

\begin{tabular}{|c|c|c|c|}
\hline & Competitors & New technology & $\begin{array}{l}\text { (i) Enhanced competition due to new } \\
\text { entrants } \\
\text { (ii) Flood of resources initially due to } \\
\text { high expectation } \\
\text { (iii)Completion by existing business } \\
\text { organizations }\end{array}$ \\
\hline & Expansion & $\begin{array}{ll}\text { Educating } \\
\text { collaborators }\end{array}$ & $\begin{array}{l}\text { (i) Importance and easiness of } \\
\text { technology } \\
\text { (ii) Product features } \\
\text { (iii) Investment by collaborators }\end{array}$ \\
\hline \multirow[t]{4}{*}{$\begin{array}{l}\text { Consumer } \\
\text { Issues }\end{array}$} & Quality & Cost & $\begin{array}{l}\text { (i) Return on price } \\
\text { (ii) Procrastination in usage } \\
\text { (iii) Doubt on expected benefits }\end{array}$ \\
\hline & Features & $\begin{array}{lr}\text { Confused } & \text { due to } \\
\text { general } & \text { purpose } \\
\text { technology } & \end{array}$ & $\begin{array}{l}\text { (i) Expected solutions in all areas of } \\
\text { society } \\
\text { (ii) Sustainability is a question } \\
\text { (iii) Questions on side effects }\end{array}$ \\
\hline & Cost & Affordability & $\begin{array}{l}\text { (i) New technology is costly } \\
\text { (ii) New technology is challenging } \\
\text { (iii) New technology is difficult } \\
\text { accept. }\end{array}$ \\
\hline & Support & Frequent service & $\begin{array}{l}\text { (i) New and hence less penetration } \\
\text { (ii) Less service centres initially } \\
\text { (iii)Services may be costly due to } \\
\text { shortage of skilled people }\end{array}$ \\
\hline \multirow[t]{2}{*}{$\begin{array}{l}\text { Environmental } \\
\text { Issues }\end{array}$} & $\begin{array}{l}\text { Environmental } \\
\text { degradation }\end{array}$ & $\begin{array}{l}\text { Complexity of the } \\
\text { system }\end{array}$ & $\begin{array}{l}\text { (i) Controlling the emission of green } \\
\text { gases is difficult process } \\
\text { (ii) Monitoring and controlling the } \\
\text { pollution is difficult } \\
\text { (iii) Industrialenvironmental pollution } \\
\text { is difficult to control }\end{array}$ \\
\hline & $\begin{array}{l}\text { Environmental } \\
\text { Improvement }\end{array}$ & Scaling & $\begin{array}{l}\text { (i) Large scale cleaning is difficult } \\
\text { throughout the globe. } \\
\text { (ii) Maintenance of cleaned } \\
\text { environment is a challengein terms of } \\
\text { energy requirement } \\
\text { (iii)All countries should involve in the } \\
\text { process. }\end{array}$ \\
\hline \multirow[t]{2}{*}{ Social Issues } & Basic problems & Commercialization & $\begin{array}{lll}\text { (i) Commercialization } & \text { of } & \text { new } \\
\text { technology is risky } & & \\
\text { (ii) Commercialization } & \text { of } & \text { new } \\
\text { technology is difficult } & & \\
\text { (iii)Commercialization } & \text { of } & \text { new } \\
\text { technology is costly. } & & \\
\end{array}$ \\
\hline & $\begin{array}{l}\text { Advanced } \\
\text { problems }\end{array}$ & $\begin{array}{l}\text { Still in experimental } \\
\text { level }\end{array}$ & $\begin{array}{l}\text { (i) Acceptance of new technology by } \\
\text { people. } \\
\text { (ii) Technology penetration is slow. } \\
\text { (iii)Side effects are yet to know. }\end{array}$ \\
\hline
\end{tabular}


International Journal of Management, Technology, and Social

\begin{tabular}{|l|l|l|l|}
\hline & Immortality & $\begin{array}{l}\text { Slow research in } \\
\text { nanotechnology }\end{array}$ & $\begin{array}{l}\text { (i) Uncertainty in anticipated results } \\
\text { (ii) Research laboratory results are } \\
\text { difficult to commercialize in expected } \\
\text { time frame } \\
\text { (iii)Anticipated negative implications } \\
\text { on immortality. }\end{array}$ \\
\hline
\end{tabular}

Table 7 : Disadvantages of Nanotechnology as Green technology

\begin{tabular}{|c|c|c|c|}
\hline Particulars & Key Attributes & Factors Affecting & Critical Constituent Elements \\
\hline \multirow[t]{3}{*}{$\begin{array}{l}\text { Organizational } \\
\text { Issues }\end{array}$} & Employees & Cost of employees & $\begin{array}{l}\text { (i) Technically trained employees are } \\
\text { costly. } \\
\text { (ii) Increase in employee turnover. } \\
\text { (iii) Employees have high bargain } \\
\text { power. }\end{array}$ \\
\hline & Infrastructure & $\begin{array}{l}\text { Risk of new technology- } \\
\text { based infrastructure } \\
\text { investment }\end{array}$ & $\begin{array}{l}\text { (i) Infrastructure investment risk } \\
\text { (ii) Infrastructure modification } \\
\text { (iii) Uncertainty in infrastructure } \\
\text { investment }\end{array}$ \\
\hline & Investment & $\begin{array}{ll}\text { Risk for } & \text { higher } \\
\text { investment } & \end{array}$ & $\begin{array}{l}\text { (i) New technologies are slow } \\
\text { penetrating } \\
\text { (ii) People acceptance is difficult. } \\
\text { (iii) Technologies which are initially } \\
\text { promising may be prohibitively } \\
\text { costly. }\end{array}$ \\
\hline \multirow[t]{4}{*}{ Business Issues } & Products & $\begin{array}{l}\text { May have hidden long } \\
\text { term affects }\end{array}$ & $\begin{array}{l}\text { (i) Side effects } \\
\text { (ii) Fail to achieve goal } \\
\text { (iii) Product features \& promotions. }\end{array}$ \\
\hline & Marketing & $\begin{array}{l}\text { Initial Cost of creating } \\
\text { awareness }\end{array}$ & $\begin{array}{l}\text { (i) New brand building cost } \\
\text { (ii) Challenge of creating awareness } \\
\text { for new products. } \\
\text { (iii) Challenge on product } \\
\text { performance. }\end{array}$ \\
\hline & Competitors & $\begin{array}{l}\text { Huge investment for } \\
\text { research \& development }\end{array}$ & $\begin{array}{l}\text { (i) Initial cost. } \\
\text { (ii) Availing patent protection. } \\
\text { (iii)Technology management }\end{array}$ \\
\hline & Expansion & Copying by others & $\begin{array}{l}\text { (i) Patent time } \\
\text { (ii) Outsourcing } \\
\text { (iii) Starting foreign subsidiaries }\end{array}$ \\
\hline \multirow[t]{3}{*}{$\begin{array}{l}\text { Consumer } \\
\text { Issues }\end{array}$} & Quality & May have side effects & $\begin{array}{l}\text { (i) Minimizing side effects } \\
\text { (ii) Precautioning during use } \\
\text { (iii) Quality control }\end{array}$ \\
\hline & Features & Fear on side effects & $\begin{array}{l}\text { (i) Check for branded products. } \\
\text { (ii) Quality control through sufficient } \\
\text { clinical trails } \\
\text { (iii) Precaution during use. }\end{array}$ \\
\hline & Cost & $\begin{array}{l}\text { Difficulty in large scale } \\
\text { operation }\end{array}$ & $\begin{array}{l}\text { (i) Investment cost } \\
\text { (ii) Maintenance cost } \\
\text { (iii) Resource cost }\end{array}$ \\
\hline
\end{tabular}


International Journal of Management, Technology, and Social

\begin{tabular}{|c|c|c|c|}
\hline & Support & $\begin{array}{l}\text { Minimum } \\
\text { support }\end{array}$ & $\begin{array}{l}\text { (i) After sales service } \\
\text { (ii) Promised quality monitoring } \\
\text { (iii) Feedback }\end{array}$ \\
\hline \multirow[t]{2}{*}{$\begin{array}{l}\text { Environmental } \\
\text { Issues }\end{array}$} & $\begin{array}{l}\text { Environmental } \\
\text { degradation }\end{array}$ & $\begin{array}{l}\text { Prolonged technology } \\
\text { breakthrough time than } \\
\text { expected }\end{array}$ & $\begin{array}{l}\text { (i) Waiting for breakthrough } \\
\text { (ii) Govt. involvement } \\
\text { (iii) Stringent rules }\end{array}$ \\
\hline & $\begin{array}{l}\text { Environmental } \\
\text { Improvement }\end{array}$ & Negative effects if any & $\begin{array}{l}\text { (i) Environmental side effects } \\
\text { (ii) Cost of environmental } \\
\text { improvement } \\
\text { (iii) Disputes in } \\
\text { responsibilities } \\
\text { lim }\end{array}$ \\
\hline \multirow[t]{3}{*}{ Social Issues } & Basic problems & Delay in realization & $\begin{array}{l}\text { (i) Long time problems are difficult } \\
\text { to tackle. } \\
\text { (ii) Delay in patent acceptance } \\
\text { (iii) Delay in availing finance for } \\
\text { investments }\end{array}$ \\
\hline & $\begin{array}{l}\text { Advanced } \\
\text { problems }\end{array}$ & $\begin{array}{l}\text { Unpredictable adverse } \\
\text { effects }\end{array}$ & $\begin{array}{l}\text { (i) Fear in adopting new technology } \\
\text { (ii) Sometimes predictions may not } \\
\text { work. } \\
\text { (iii) Global regulations may hider } \\
\text { firms performance. }\end{array}$ \\
\hline & Immortality & $\begin{array}{l}\text { Negative aspects of } \\
\text { immortality } \\
\text { /deathlessness }\end{array}$ & $\begin{array}{l}\text { (i) Aging } \\
\text { (ii) Antiaging } \\
\text { (iii) Stagnated growth \& challenges. }\end{array}$ \\
\hline
\end{tabular}

\section{CONCLUSION :}

In this paper, we have analysed the Nanotechnology as green technology using ABCD analysis framework. 64 affecting factors of nanotechnology as green technology and 192 critical constituent elements are identified in the analysis under the four constructs advantages, benefits, constraints, and disadvantages. Thus, nanotechnology as a technology for sustaining and improving green environment has many opportunities and challenges to solve organizational problems, business problems, customers problems, environmental problems, and societal problems in order to change the life style and health of the human beings and hence considered as boon of human species to grow, expand, and enjoy the life without may varies in future days. Such interpretation of nanotechnology as a green technology through its affecting factors and critical constituent elements are going to interpret the consequences of this new technology favourable continuation of human life in this universe.

\section{REFERENCES :}

[1] Aithal, P. S., (March 2015). Concept of Ideal Business \& Its Realization Using EBusiness Model. International Journal of Science and Research (IJSR), 4(3), 1267 1274. ISSN 2319-7064, DOI :http://doi.org/10.5281/zenodo.61648.

[2] Aithal, P. S. and Shubhrajyotsna Aithal (2018). Study of various General-Purpose Technologies and Their Comparison towards developing Sustainable Society. International Journal of Management, Technology, and Social Sciences (IJMTS), 3(2), 16-33.

[3]Aithal, P. S. (2016). Study on ABCD Analysis Technique for Business Models, Business strategies, Operating Concepts \& Business Systems, International Journal in Management and Social Science, 4(1), 98115. DOI :http://doi.org/10.5281/zen odo.161137.

[4]Aithal, P. S. and Suresh Kumar, P. M. (2015). Applying SWOC Analysis to an Institution 
of Higher Education. International Journal of Management, IT and Engineering (IJMIE), 5(7), 231-247. DOI: http://doi.org/10.5281/zenodo.163425.

[5] Madhushree, L. M., Revathi, R., Anil Kumar, Aithal, P.S., (2018). Business Strategies of Top Indian IT Company: Mindtree. International Journal of Case Studies in Business IT and Education (IJCSBE), 2(1), 22-36. DOI: http://dx.doi.org/10.5281/zenedo.1249871.

[6] Aithal, P. S., Shailashree, V. T., Suresh Kumar, P. M. (2015). A New ABCD Technique to Analyze Business Models \& Concepts, International Journal of Management, IT and Engineering (IJMIE), 5(4), 409-423. DOI :http://doi.org/10.5281/zenodo.61652.

[7] Gupta, A. (2013). Environment \& PEST analysis: an approach to external business environment. International Journal of Modern Social Sciences, 2(1), 34-43.

[8] Aithal P. S., (2017). Impact of Domestic, Foreign, and Global Environments on International Business Decisions of Multinational Firms: A Systematic Study. International Journal of Management, Technology, and Social Sciences (IJMTS), 2(2), 57-73. DOI: http://dx.doi.org/10.5281/zenodo.1067103.

[9] Aithal, P. S., Shailashree, V. T., \& Suresh Kumar, P. M. (2015). Application of ABCD Analysis Model for Black Ocean Strategy. International Journal of Applied Research (IJAR), 1(10), 331-337. DOI: http://doi.org/ 10.5281/zenodo.163424.

[10] Aithal, P. S., Shailashree, V. T., \& Suresh Kumar P. M., (2016). ABCD analysis of Stage Model in Higher Education. International Journal of Management, IT and Engineering (IJMIE), 6(1), 11-24. DOI: http://doi.org/10.5 281/zenodo.154 233.

[11] Aithal, P. S., Shailashree, V. T., \& Suresh Kumar, P. M. (2016). Analysis of NAAC Accreditation System using ABCD framework. International Journal of Management, IT and Engineering (IJMIE), 6(1), 30-44. DOI: http://doi.org/10. 5281/zenodo.154272.

[12] Aithal, P. S., Shailashree, V. T., \& Suresh Kumar, P. M. (2016). Application of ABCD Analysis Framework on Private University System in India. International Journal of Management Sciences and Business Research (IJMSBR), 5(4), 159170. DOI :http://doi.org/10.5281/zenodo.161111.

[13] Aithal, P. S., Shailashree, V. T., \& Suresh Kumar, P. M. (2016). The Study of New National Institutional Ranking System using ABCD Framework, International Journal of Current Research and Modern Education (IJCRME), 1(1), 389-402. DOI :http://doi.org/10.5281/zenodo.161077.

[14] Aithal, S., \& Aithal, P. S. (2016). ABCD analysis of Dye doped Polymers for Photonic Applications, IRA-International Journal of Applied Sciences, 4 (3), 358378. DOI: http://dx.doi.org/10. 21013/j as.v4.n3.p1.

[15] Aithal, P. S., Shailashree, V. T. \& Suresh Kumar, P. M., (2016). Analysis of ABC Model of Annual Research Productivity using ABCD Framework. International Journal of Current Research and Modern Education (IJCRME), 1(1), 846-858. DOI :http://doi.org/10.5281/ zenodo.62022.

[16] Varun Shenoy, \& Aithal P. S., (2016). ABCD Analysis of On-line Campus Placement Model, IRA-International Journal of Management \& Social Sciences, 5(2), 227-244. DOI: http://dx.doi.org/10.21013/jmss .v5.n2.p3.

[17] Aithal, P. S., Shailashree V. T. \& Suresh Kumar P.M. (2016). Factors \& Elemental Analysis of Six Thinking Hats Technique using ABCD Framework. International Journal of Advanced Trends in Engineering and Technology (IJATET), 1(1), 85-95. DOI : http://doi.org/10.5281/zenodo.240 $\underline{259}$. 
[18] Aithal, P. S. \& Suresh Kumar, P. M. (2016). CCE Approach through ABCD Analysis of 'Theory A' on Organizational Performance. International Journal of Current Research and Modern Education (IJCRME) 1(1), 169-185. DOI: http://dx.doi.org/10. 5281/zenodo.164704.

[19] Aithal, P. S. (2017). ABCD Analysis of Recently Announced New Research Indices. International Journal of Management, Technology, and Social Sciences (IJMTS), 2(1), 65-76. DOI: http://doi.org/10.5281/ zenodo. 583644.

[20] Aithal, P. S. (2017). Factor Analysis based on ABCD Framework on Recently Announced New Research Indices, International Journal of Management, Technology, and Social Sciences (IJMTS), 1(1), 82-94. DOI: http://dx.doi.org/1 $\underline{0.5281 / \text { zenodo.584105. }}$.

[21] Aithal, P. S., (2017).ABCD Analysis as Research Methodology in Company Case Studies. International Journal of Management, Technology, and Social Sciences (IJMTS), 2(2), 40-54. DOI: http://dx.doi.org/10.5 281/zenodo.891621.

[22] Aithal, Architha., Aithal, P. S. (2017). ABCD Analysis of Task Shifting-An optimum Alternative Solution to Professional Healthcare Personnel Shortage. International Journal of Health Sciences and Pharmacy (IJHSP), 1(2), 3651. DOI: http://dx.doi.org/10.5281/zeno do.1038975.

[23] Varun Shenoy \& Aithal, P. S., (2017). Quantitative ABCD Analysis of IEDRA Model of Placement Determination. International Journal of Case Studies in Business, IT and Education (IJCSBE), 1(2), 103-113. DOI: http://dx.doi.org/10.5281/z enodo.1133691.

[24] Keerthan Raj, \& Aithal, P. S. (2018 March). Generating Wealth at the Base of the Pyramid - A Study using ABCD Analysis Technique. International Journal of Computational Research and Development
(IJCRD), $\quad 3(1), \quad 68-76 . \quad$ DOI :http://doi.org/10.5281/zenodo.1205586.

[25] Shubhrajyotsna Aithal and Aithal P. S., (2016), ABCD analysis of Dye doped Polymers for Photonic Applications, IRAInternational Journal of Applied Sciences, 4(3), 358-378. DOI :http://doi.org/10.5281/zenodo.155103. DOI :http://dx.doi.org/10.21013/jas.v4.n3.p1.

[26] Krishna Prasad \& Aithal P. S. (2018). ABCD Analysis of Fingerprint Hash Code, Password and OTP based Multifactor Authentication Model. Saudi Journal of Business and Management Studies, 3(1), 65-80.

DOI: http://doi.org/10.21276/sjbms.2018.3.1.10.

[27] Keerthan Raj, \& Aithal, P. S. (2018). Generating Wealth at the Base of the Pyramid - A Study using ABCD Analysis Technique. International Journal of Computational Research and Development (IJCRD), 3(1), 68-76. DOI :http://doi.org/10.5281/zenodo.1205586.

[28] Architha Aithal \& A. R. Shabaraya (2018). Users Perspectives on Online Pharmacy Model. International Journal of Health Sciences and Pharmacy (IJHSP), 2(1), 2936. DOI: http://dx.doi.org/10.5281/zenodo. $\underline{1292790 .}$.

[29] Narula, A.V., \& Aithal, P. S. (2018).Employability Skill traits Management Quotient [ESMQ] - A Conceptual Model Proposal. International Journal of Applied Engineering and Management Letters (IJAEML), 2(1), 1-30. DOI: $\quad$ http://dx.doi.org/10.5281/zenod $\underline{0.1156138}$

[30] Aithal, P. S. \& Suresh Kumar, P. M. (2016). Opportunities and Challenges for Private Universities in India. International Journal of Management, IT and Engineering (IJMIE), 6(1), 88-113. DOI :http://doi.org/10.5281/zenodo. 161157.

[31] Padmanabha Shenoy, \& Aithal, P. S., (2016). A Study on History of Paper and 
possible Paper Free World. International Journal of Management, IT and Engineering (IJMIE), 6(1), 337-355. DOI :http://doi.org/10.5281/zenodo. 161141.

[32] Aithal, P.S., (2015). Comparative Study on MBA Programmes in Private \& Public Universities - A case study of MBA programme plan of Srinivas University, International Journal of Management Sciences and Business Research (IJMSBR), 4(12), 106-122.

DOI:http://doi.org/10.5281/z enodo.163884.

[33] Aithal P. S., \&Shubhrajyotsna Aithal (2016). Impact of On-line Education on Higher Education System. International Journal of Engineering Research and Modern Education (IJERME), 1(1), 225$235 . \quad$ DOI :http://doi.org/ 10.5281/zenodo.161113.

[34] Aithal P. S., and Suresh Kumar P. M., (2016). Analysis of Choice Based Credit System in Higher Education. International Journal of Engineering Research and Modern Education (IJERME), 1(1), 278$284 . \quad$ DOI :http://doi.org/ 10.5281/zenodo.161046.

[35] Varun Shenoy and Aithal, P. S., (2016). Changing Approaches in Campus Placements - A new futuristic Model, International Journal of Scientific Research and Modern Education (IJSRME), 1(1), 766
- 776. DOI :http://doi.org /10.5281/zeno do.160966.

[36] Prithi Rao, and Aithal, P.S. (2016). Green Education Concepts \& Strategies in Higher Education Model, International Journal of Scientific Research and Modern Education (IJSRME), 1(1), 793-802. DOI :http://doi.org/ 10.5281/zenodo.160877.

[37] Aithal, P. S. \& Shubhrajyotsna Aithal (2016). Ekalavya Model of Higher Education - an Innovation of IBM's Big Data University. International Journal of Current Research and Modern Education (IJCRME), 1(2), 190-205. DOI: http://dx.doi.org/10.5281/ZENODO.1 98704.

[38] Aithal, P. S. and Aithal, Shubhrajyotsna, (2016). Opportunities \& Challenges for Green Technology in 21st Century. International Journal of Current Research and Modern Education (IJCRME), 1(1), 818-828.DOI http://doi.org/10.5281/zenodo.62020.

[39] Nanotechnology and Life Cycle Assessment, Washington DC, 2-3 October 2006.

http://www.nanotechproject.org/file_downl oad/files/NanoLCA 3.07.pdf referred on 03/09/2018. 